bioRxiv preprint doi: https://doi.org/10.1101/2020.04.26.062927; this version posted April 28, 2020. The copyright holder for this preprint (which

was not certified by peer review) is the author/funder, who has granted bioRxiv a license to display the preprint in perpetuity. It is made available under aCC-BY-NC-ND 4.0 International license.

\title{
Coevolution of song and egg coloration: multimodal mating signals?
}

2

3

4

5

6

7

Silu Wang

University of California, Berkeley

Email: siluwang.evo@gmail.com 


\section{Introduction}

Reproductive trait divergence is crucial for speciation because these traits frequently underpin barriers to gene flow (Koski and Ashman 2016; Pfennig 2016). A very puzzling reproductive trait divergence exists in tinamou egg coloration. Tinamiformes (common name: tinamou) is the most species-rich order of Palaeognathae, containing 48 extant species (Figure 1) (Cabot 1992; Davies 2002). Tinamous are mostly dull in plumage, but various species of tinamous lay brightly colored eggs ranging from brilliant magenta, to pink, purple, turquoise, and olive green (Figure 1) (Cabot 1992). Despite its evolutionary importance, tinamou egg color divergence remains a mystery due to the cryptic and elusive plumage and behavior of the birds (Cabot 1992; Davies 2002).

Two main hypotheses have been made about evolutionary mechanism of egg color divergence: (1) aposematism hypothesis (Swynnerton 1916) states that eggs are brightly colored to warn predators of their distastefulness; (2) mating signaling hypothesis, which predicts that egg color are employed for mating recognition and attraction (Weeks 1973; Brennan 2010). The aposematic function of egg coloration was thought to be unlikely, because egg predators are nocturnal mammals or reptiles that prioritize chemical cues over visual cues (Skutch 1966; Cabot 1992; Brennan 2010). The mating signal hypothesis is plausible considering the ecology and mating systems of tinamou species. In most tinamou species, males collect and incubate eggs laid by multiple females (Cabot 1992; Davies 2002). An initial female is attracted by distinctive songs to the nest guarded a male. If mating occurs, the female lays eggs in the nest and leaves before other females come and lay more eggs that are all incubated by the same male (Cabot 1992; Davies 2002). The colors of the existing eggs in the nest could signal intra-specific mate identity to prevent costly hybridization. Such alternative "mating channel" might be especially favored when the plumage is adapted for camouflage (Cabot 1992; Davies 2002). If egg colors of different tinamou species is adapted for mate recognition, reinforcement (Dobzhansky 1937; Mayr 1942; Liou and Price 1994; Servedio 2000) could have driven egg color divergence among different tinamou species. 
Here I test the mating signal hypothesis of tinamou egg color divergence. If tinamou egg colors are employed as a species-specific mate recognition signal, it should coevolve with other mate-recognition signals as the tinamou lineages diverged. Songs of tinamous are known to be important and highly divergent mating signals among species (Cabot 1992; Bertelli and Tubaro 2002; Laverde-R and Cadena 2014). In addition to songs, multimodality of mating signals is selected for efficient mate-recognition in complex environment (Partan and Marler 1999; Rowe 1999; Secondi et al. 2015; Halfwerk et al. 2019). When glamorous plumage is costly due to increased predation risk, alternative signal modality such as egg coloration could be favored. Since the birds themselves (instead of the eggs) tend to attract nest predation (Brennan 2010), egg coloration could be employed to fulfill signal multimodality, bypassing the evolutionary constraints for plumage. To investigate this possibility, here I test whether egg coloration coevolved with songs as tinamou species diverged.

\section{Methods}

To test coevolution of egg color and song in tinamous, I tested the association of songs and egg colors among 32 tinamou species in R (Hothorn et al. 2008). Tinamou egg coloration data was extracted from existing tinamou egg color analysis (Schläpfer 2017), which quantified coloration of the eggs from 32 tinamou species (Figure 1). Since tinamou egg colors are known to decay over the course museum storage, tinamou nest photos are used to best represent the functional egg color (Schläpfer 2017). Briefly, for each species, the egg coloration reflected in hue, chroma, and luminance in the CIELAB color space was quantified (Schläpfer 2017).

Song data was acquired from a previous study (Bertelli and Tubaro 2002), in which four song variables from 40 tinamou species were quantified: maximum frequency $(\mathrm{Hz})$, minimum frequency $(\mathrm{Hz})$, emphasized frequency (frequency of the note with highest amplitude in the song, $\mathrm{Hz}$ ), and bandwidth (the difference between maximum and minimum frequency, $\mathrm{Hz}$ ). Because these variables are correlated (Figure S1), I used principle component analysis (PCA) to generate $\mathrm{PC} 1$ that captured $81.2 \%$ of the variation in song data. Four song variables were shifted to center around zero and scaled to unit variance preceding PCA.

I tested whether egg hue, chroma, and luminance is respectively associated with song PC1 among tinamous, accounting for the potential confounding phylogenetic signals. I used the Tinamidae phylogenetic tree inferred with both molecular (1143 bp mitochondrial and $1145 \mathrm{bp}$ nuclear) and morphological characters (237 characters) (Bertelli and Porzecanski 2004), in which the polytomy was resolved with additional morphological and life history traits (Bertelli 2017). I first tested if there is phylogenetic signal (Pagel 1999) in each of the egg color variable with phytools (Revell 2012). If no significant phylogenetic signal was identified, I ran permutation test of independence between song PC1 and the egg color variable with coin package. If there is significant $(p<0.05)$ phylogenetic signal, I first calculated independent contrasts of egg colors and song PC1 with Ape (Paradis and Schliep 2019), then executed independence test. To correct for multiple hypotheses testing, I conducted False Discovery Rate correction (Benjamini and Hochberg 1995) to correct for three tests.

\section{Results}

I found a significant association between tinamou songs and egg luminance. Song PC1 effectively represents variation among the song variables in tinamous (Figure 2A). Significant phylogenetic signal was observed in egg hue $(\mathrm{K}=0.43, p=0.049)$ and chroma $(\mathrm{K}=0.96, p=$
$0.001)$, but not in luminance $(\mathrm{K}=0.37, p=0.17)$. The tinamou song PC1 was significantly 
bioRxiv preprint doi: https://doi.org/10.1101/2020.04.26.062927; this version posted April 28, 2020. The copyright holder for this preprint (which was not certified by peer review) is the author/funder, who has granted bioRxiv a license to display the preprint in perpetuity. It is made available under aCC-BY-NC-ND 4.0 International license.

associated with egg luminance $\left(Z=-2.42, p=0.015\right.$, $p_{\text {corrected }}=0.045$; Figure $\left.2 \mathrm{~B}\right)$, but not with egg hue $\left(Z=0.34, p=0.72, p_{\text {corrected }}=0.75\right)$, nor with egg chroma $\left(Z=-0.32, p=0.75\right.$, $p_{\text {corrected }}=$ $0.75)$.

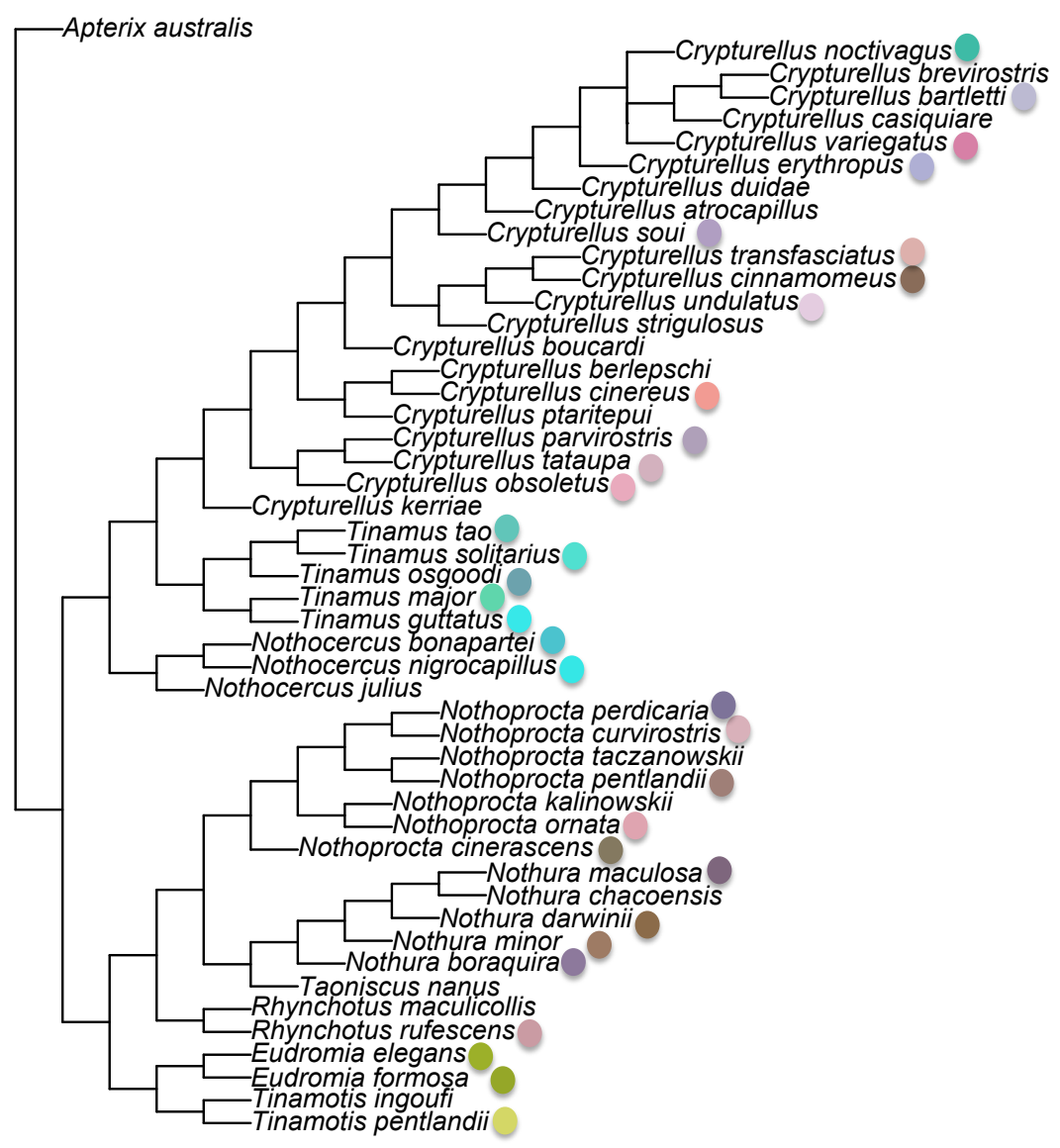

Figure 1 Phylogeny of tinamous and variability in egg colour The Tinamidae phylogeny was 

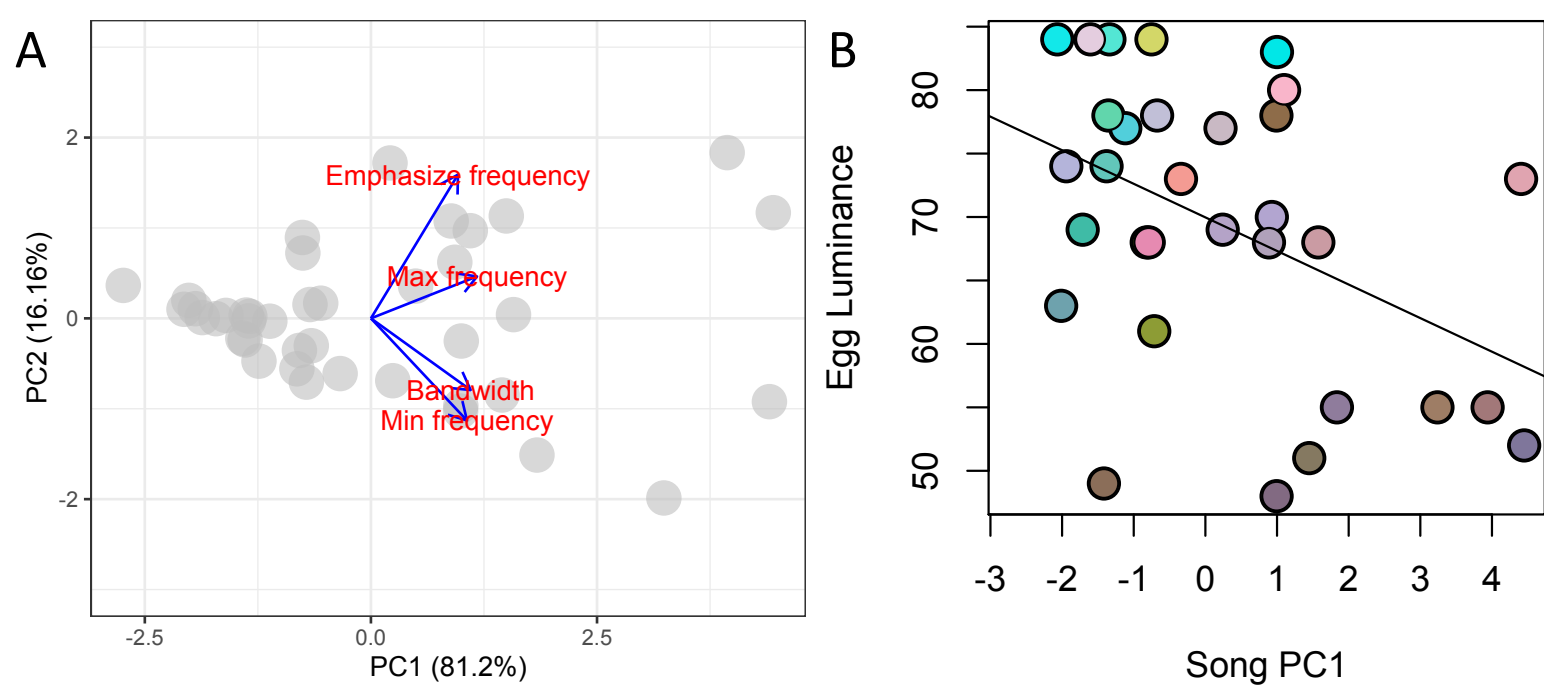

Figure 2 Principal components analysis of tinamou song characteristics and their association with egg colour (A) a biplot of the song PCA with blue vectors representing projected song variables (labeled in red). (B) There is significant association between egg luminance and song PC1 (permutation test of independence, $\mathrm{Z}=-2.42, \mathrm{R}^{2}=0.20, p=0.015$, $\left.p_{\text {corrected }}=0.045\right)$. The color of dots represent the RGB color of the eggs of each species. 
The coevolution of tinamou song and egg coloration supports the hypothesis that tinamou egg coloration is an alternative mating signal (Weeks 1973; Brennan 2010). Egg colors and song could be divergently selected as multimodal mate-recognition signals among tinamou species with similar appearance. When the plumage modality is constrained by anti-predation adaptation, egg coloration could be opportunistically adopted to fulfill mating signal multimodality. This study sheds light on the evolution of multimodal sexual signals that bypasses natural selection for plumage camouflage in the most species-rich order of Palaeognathae.

Egg coloration could be both a pre- and post-mating signals in tinamous. In many other bird species, egg color is known to be post-mating signals indicating female quality and to influence male incubation and promiscuity (Soler et al. 2005; English 2009). In tinamous, egg coloration can also function as premating signal as well, received by females. For most of the tinamou species, males guard and incubate the eggs laid by multiple females (Cabot 1992). The coloration of the existing eggs in the nest could be a mating signal received by the upcoming females to the nest. As premating signals, egg colors could reflect mate species identity (Sætre et al. 1997; Servedio and Noor 2003; Secondi et al. 2015) and mate quality, stimulating female mate choice copying (Dugatkin 1992; Gibson and Höglund 1992).

Then why do tinamous adopt egg colors as mating signals in addition to songs? Songs that are important mating signals in tinamous (Bertelli and Tubaro 2002) and are involved in duetting between mating partners (Boesman et al. 2018). However, such acoustic modality is usually insufficient for mate communication in complex environment, thus signal redundancy or multimodality is further selected for mate communication to alleviate mate-searching effort and/or hybridization (Partan and Marler 1999; Rowe 1999; Uy et al. 2008; Secondi et al. 2015). Plumage patterning and coloration are frequently adopted as inter-specific and intra-specific mating signals at a finer spatial scale in birds (Uy et al. 2008, 2009; Seddon et al. 2013). However, most tinamou species exhibited cryptic plumage as an adaption for anti-predatory camouflage (Cabot 1992; Davies 2002). Sexual selection for plumage elaboration would compromise the adaptation for camouflage favored by natural selection. Egg coloration can be an alternative modality of mate signaling enrichment towards mate-searching refinement, bypassing the conflict between natural and sexual selection.

Reinforcement may have driven tinamou egg color and song divergence among sympatric or parapatric tinamou species. Many closely-related tinamou species demonstrate historical and/or contemporary range overlap (Cabot 1992), suggesting potential sympatry in the course of their speciation history. Ecological and/or intrinsic incompatibility among diverged lineages leads to reduced hybrid fitness, which in turn select for premating signal divergence to avoid costly hybridization (Dobzhansky 1940; Mayr 1942; Liou and Price 1994; Servedio 2000). Mating signal multimodality is needed to ensure mate recognition in complex heterospecific environment (Uy et al. 2008; Secondi et al. 2015). The divergence of tinamou song and egg coloration could jointly reduce heterospecific reproductive efforts among sympatric tinamou species. Closely related tinamou species that are currently in allopatry could still harbor footprints of historical character displacement of egg colors formed at historical sympatry. For example, the three closely related species Crypturellus erythropus, C. notivagus, C. variegatus demonstrate distinct egg coloration, which may have been driven by reinforcement at historical sympatry. The historical geography and ecology around the speciation event is most relevant to recreate the potential egg color displacement. However, it is challenging to retrieve such 
information, because the splits among these three tinamou species have long passed ( $\sim 34 \%$ sequence divergence).

The genetic underpinning of such song and egg color association is unclear. The simplest genetic mechanism underlying such association is pleiotropy (one gene affecting multiple traits) (Fisher 1930; Williams 1957). For example, the pleiotropic foraging gene underpins the association among social behavior and lifehistory traits in natural populations (de Belle et al. 1989; Mery et al. 2007; Wang and Sokolowski 2017). There might be an omnipotent pleiotropic gene linking egg coloration, songs, among many other traits. If so, a wide array of traits underpinned by such pleiotropy are expected to coevolve. However, the association between song and egg color of tinamous is fairly specific: only luminance (but not hue or chroma) of the egg color is associated with tinamou songs. Such specificity in song-egg-color association is consistent with mating signal multimodality in which specifically signals were coupled among modalities (Gilliard 1956; Partan and Marler 1999; Hebets and Papaj 2005). Various signal modality can be underpinned by different genes and selected to coevolve as multimodal signals. Future investigation of genetic underpinnings of tinamou song and egg colors would further shed light on this interesting co-divergence. In sum, the results presented herein are concordant with the hypothesis that egg coloration in tinamous serves as mating signals either for mate attraction or mate species-recognition.

\section{Acknowledgement}

Thank Dahong Chen, Patricia Brennan, Daniel Hanley, Julia Clarke, and Jonathan Rolland for helpful discussions. Thank Ken Thompson, Dahong Chen, and Jonathan Rolland for comments on the manuscript.

\section{Reference}

Benjamini, Y., and Y. Hochberg. 1995. Controlling the False Discovery Rate: A Practical and Powerful Approach to Multiple Testing. J. R. Stat. Soc. Ser. B 57:289-300.

Bertelli, S. 2017. Advances on tinamou phylogeny: an assembled cladistic study of the volant palaeognathous birds. Cladistics 33:351-374.

Bertelli, S., and A. L. Porzecanski. 2004. Tinamou (tinamidae) systematics: a preliminary combined analysis of morphology and molecules. Ornitol. Neotrop. 15:293-299.

Bertelli, S., and P. L. Tubaro. 2002. Body mass and habitat correlates of song structure in a primitive group of birds. Biol. J. Linn. Soc. 77:423-430.

Boesman, P., O. Claessens, T. V. V. Costa, V. Pelletier, J. Ingels, and A. Renaudier. 2018. Songs of Rusty Tinamou Crypturellus brevirostris and duetting in Crypturellus species. Bull. Br. Ornithol. Club 138:69-78.

Brennan, P. L. R. 2010. Clutch predation in great tinamous Tinamus major and implications for the evolution of egg color. J. Avian Biol. 41:419-426.

Cabot, J. 1992. Tinamiformes. Pp. 112-144 in D. Hoyo, A. Elliot, and J. Sargatal, eds. Handbook of birds of the world. Barcelona.

Davies, S. J. J. F. 2002. Ratities and tinamous. Tinamidae, Rheidae, Dromaiidae, Casuariidae, Apterygidae, Struthionidae. Oxford Univ. Press, New York.

de Belle, J. S., A. J. Hilliker, and M. B. Sokolowski. 1989. Genetic localization of foraging (for): a major gene for larval behavior in Drosophila melanogaster. Genetics 123:157-164.

Dobzhansky, T. 1937. Genetics and the Origin of Species. Columbia University Press, New 
York.

Dobzhansky, T. 1940. Speciation as a Stage in Evolutionary Divergence. Am. Nat. 74:312-321.

Dugatkin, L. A. 1992. Sexual Selection and Imitation: Females Copy the Mate Choice of Others. Am. Nat. 139:1384-1389.

English, P. 2009. Why is a robin's egg blue? Exploring the evolution of egg colour in birds. Queen's University.

Fisher, R. A. 1930. The genetical theory of natural selection. Oxford Univ. Press, New York, NY.

Gibson, R., and J. Höglund. 1992. Copying sexual selection. Trends Ecol. Evol. 7:229-232.

Gilliard, E. T. 1956. Bower Ornamentation versus Plumage Characters in Bower-Birds. Auk 73:450-451.

Halfwerk, W., J. Varkevisser, R. Simon, E. Mendoza, C. Scharff, and K. Riebel. 2019. Toward testing for multimodal perception of mating signals. Front. Ecol. Evol. 7:1-7.

Hebets, E. A., and D. R. Papaj. 2005. Complex signal function: Developing a framework of testable hypotheses.

Hothorn, T., K. Hornik, M. A. van De Wiel, and A. Zeileis. 2008. Implementing a Class of Permutation Tests COIN Package. J. Stat. Softw. 28:1-23.

Koski, M. H., and T. L. Ashman. 2016. Reproductive character displacement and environmental filtering shape floral variation between sympatric sister taxa. Evolution (N. Y). 70:26162622.

Laverde-R., O., and C. D. Cadena. 2014. Taxonomy and conservation: A tale of two tinamou species groups (Tinamidae, Crypturellus). J. Avian Biol. 45:484-492.

Liou, L. W., and T. D. Price. 1994. Speciation by Reinforcement of Premating Isolation. Evolution (N. Y). 48:1451-1459.

Mayr, E. 1942. Systematics and the origin of species. Columbia University Press, New York.

Mery, F., A. T. Belay, A. K. C. So, M. B. Sokolowski, and T. J. Kawecki. 2007. Natural polymorphism affecting learning and memory in Drosophila. Proc. Natl. Acad. Sci. U. S. A. 104:13051-13055.

Pagel, M. 1999. Inferring the historical patterns of biological evolution. Nature 401:877-884.

Paradis, E., and K. Schliep. 2019. Ape 5.0: An environment for modern phylogenetics and evolutionary analyses in R. Bioinformatics 35:526-528.

Partan, S., and P. Marler. 1999. Communication goes multimodal. Science (80-. ). 283:12721273.

Pfennig, K. S. 2016. Reinforcement as an initiator of population divergence and speciation. Curr. Zool. 62:145-154.

Revell, L. J. 2012. phytools: An R package for phylogenetic comparative biology (and other things). Methods Ecol. Evol. 3:217-223.

Rowe, C. 1999. Receiver psychology and the evolution of multicomponent signals. Anim. Behav., doi: 10.1006/anbe.1999.1242.

Sætre, G. P., T. Moum, S. Bureš, M. Král, M. Adamjan, and J. Moreno. 1997. A sexually selected character displacement in flycatchers reinforces premating isolation. Nature 387:589-592.

Schläpfer, K. 2017. The colorful eggs of the tinamous.

Secondi, J., G. Rodgers, F. Bayle, S. Sourice, and M. Théry. 2015. Mate preference, species recognition and multimodal communication in heterogeneous environments. Evol. Ecol. 29:217-227. 
Seddon, N., C. A. Botero, J. A. Tobias, P. O. Dunn, H. E. A. MacGregor, D. R. Rubenstein, J. A. C. Uy, J. T. Weir, L. A. Whittingham, and R. J. Safran. 2013. Sexual selection accelerates signal evolution during speciation in birds. Proc. R. Soc. B Biol. Sci. 280:20131065.

Servedio, M. R. 2000. Reinforcement and the genetics of nonrandom mating. Evolution (N. Y). 54:21-29.

Servedio, M. R., and M. A. Noor. 2003. The role of reinforcement in speciation: Theory and data. Annu. Rev. Ecol. Syst. 34:339-364.

Skutch, A. F. 1966. A breeding bird census and nesting success in Central America. Ibis (Lond. 1859). 108:1-16.

Soler, J. J., J. Moreno, J. M. Avilés, and A. P. Møller. 2005. Blue and green egg-color intensity is associated with parental effort and mating system in passerines: support for the sexual selection hypothesis. Evolution (N. Y). 59:636-644.

Swynnerton, C. F. M. 1916. On the Coloration of the Mouths and Eggs of Birds: On the Coloration of Eggs. Ibis (Lond. 1859). 58:529-606.

Uy, J. A. C., R. G. Moyle, and C. E. Filardi. 2008. Plumage and song differences mediate species recognition between incipient flycatcher species of the Solomon Islands. Evolution (N. Y). 63:153-164.

Uy, J. A. C., R. G. Moyle, C. E. Filardi, and Z. A. Cheviron. 2009. Difference in Plumage Color Used in Species Recognition between Incipient Species Is Linked to a Single Amino Acid Substitution in the Melanocortin-1 Receptor. Am. Nat. 174:244-254.

Wang, S., and M. B. Sokolowski. 2017. Aggressive behaviours, food deprivation and the foraging gene. R. Soc. open Sci. 4:170042.

Weeks, S. . 1973. The behavior of the red-winged tinamou (Rhynchotus rufescens). Zoologica 58:13-40.

Williams, G. C. 1957. Pleiotropy, Natural Selection, and the Evolution of Senescence. Evolution (N. Y). 11:398-411.

\section{Supplementary information}



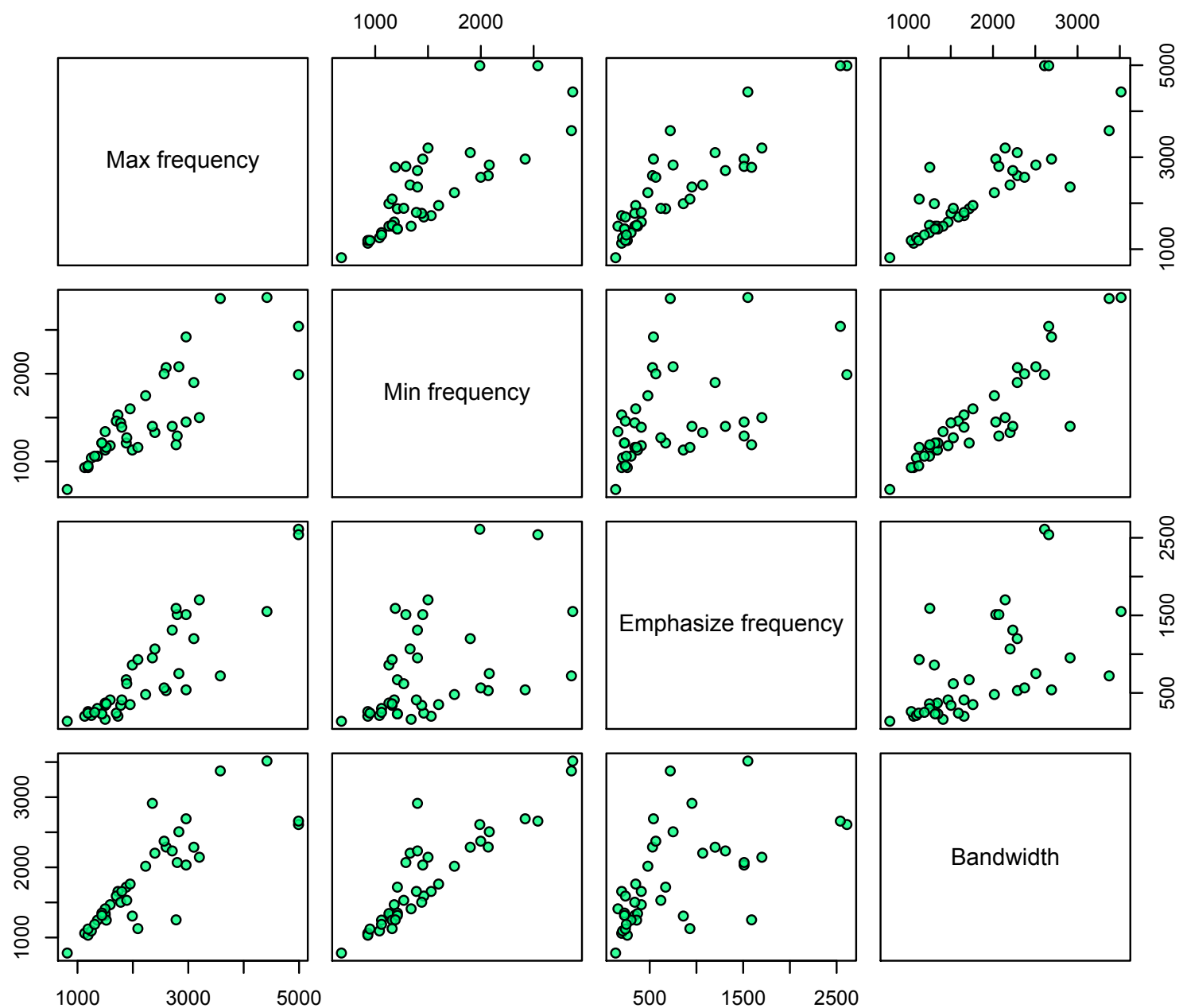

Figure S1 Scatterplots showing correlations among the four song variables. 